\title{
A Comparative Study on Channel Coding Scheme for Underwater Acoustic Communication
}

\author{
Mustafa Sami Ahmed ( $\nabla$ mustafa_sami87@yahoo.com ) \\ Universiti Tun Hussein Onn Malaysia https://orcid.org/0000-0002-3741-7091 \\ Nor Shahida Mohd Shah \\ Universiti Tun Hussein Onn Malaysia
}

\section{Research Article}

Keywords: Channel coding scheme, underwater acoustic, Convolutional, LDPC, Turbo, Polar, t-distribution

Posted Date: September 13th, 2021

DOl: https://doi.org/10.21203/rs.3.rs-826429/v1

License: (9) This work is licensed under a Creative Commons Attribution 4.0 International License. Read Full License 


\title{
A comparative study on Channel Coding Scheme for underwater acoustic
} communication

\author{
Mustafa Sami Ahmed ${ }^{1}$, Nor Shahida Mohd Shah ${ }^{2}$ \\ (mustafa_sami87@yahoo.com), (shahida@uthm.edu.my) \\ ${ }^{1}$ Departmant of Communication Engineering, Faculty of Electrical and Electronic \\ Engineering, Universiti Tun Hussein Onn Malaysia, Parit Raja, Batu Pahat, 86400, \\ Johor, Malaysia.
}

\begin{abstract}
There are various challenges in underwater acoustic communication (UWA) however bit error rate (BER) is considered as the main challenge as it significantly affects the UWA communication. In this paper, different coding schemes such as Convolution, Turbo, LDPC, and Polar coding based on the t-distribution noise channel are investigated, and binary phase-shift keying (BPSK) modulation with a code rate of $1 / 2$ has considered in the evaluation and analyses. The evaluation of these channel coding schemes is performed based on BER, computational complexity as well as latency. The results have shown the outperform of polar coding in UWA over other channel coding schemes as it has lower BER and lower computational complexity.
\end{abstract}

Keywords: Channel coding scheme; underwater acoustic; Convolutional; LDPC;

Turbo; Polar; t-distribution.

\section{Introduction}

Under water explorations, subsea resource extractions, pollution monitoring, national defence missions etc. are being critically analysed by underwater acoustic communications (UWAC) and networking technologies. The applications of UWAC have attracted increased interests as the oceans are explored as a sustainable source of food and energy [1], [2],[3]. Communications across shallow seawater channels are characterized by high multipath, high noise level, strong Doppler and other complex random transmission features. These complicate underwater communication (UWC) in terms of reliability and a low data rate compared with wireless communication [4].

Recent research focus on acoustic communications has sought to address issues such as reliability, latency, and bandwidth efficiency, where adaptation to the changing propagation conditions is the main focus [2]. In the systems' designs, the nature of the acoustic propagation forces trade-offs on the various issues depending 
on the requirements. For instance, reliability is a trade-off for a high data rate while latency is traded for full reliability. Moreover, a low computational complexity is required to achieve a high-speed data rate system. Thus, in channel coding, computational complexity and reliability are compromised with respect to each other depending on the systems' design. Figure 1 demonstrates the trade-off between reliability, latency and computational complexity considered in channel coding techniques. These channel trade-offs and constraints are challenging; however, acoustic channel exhibit peculiar challenges and solutions. This paper considers a user requirement channel coding scheme for UWAC system of lengthy message transmission.

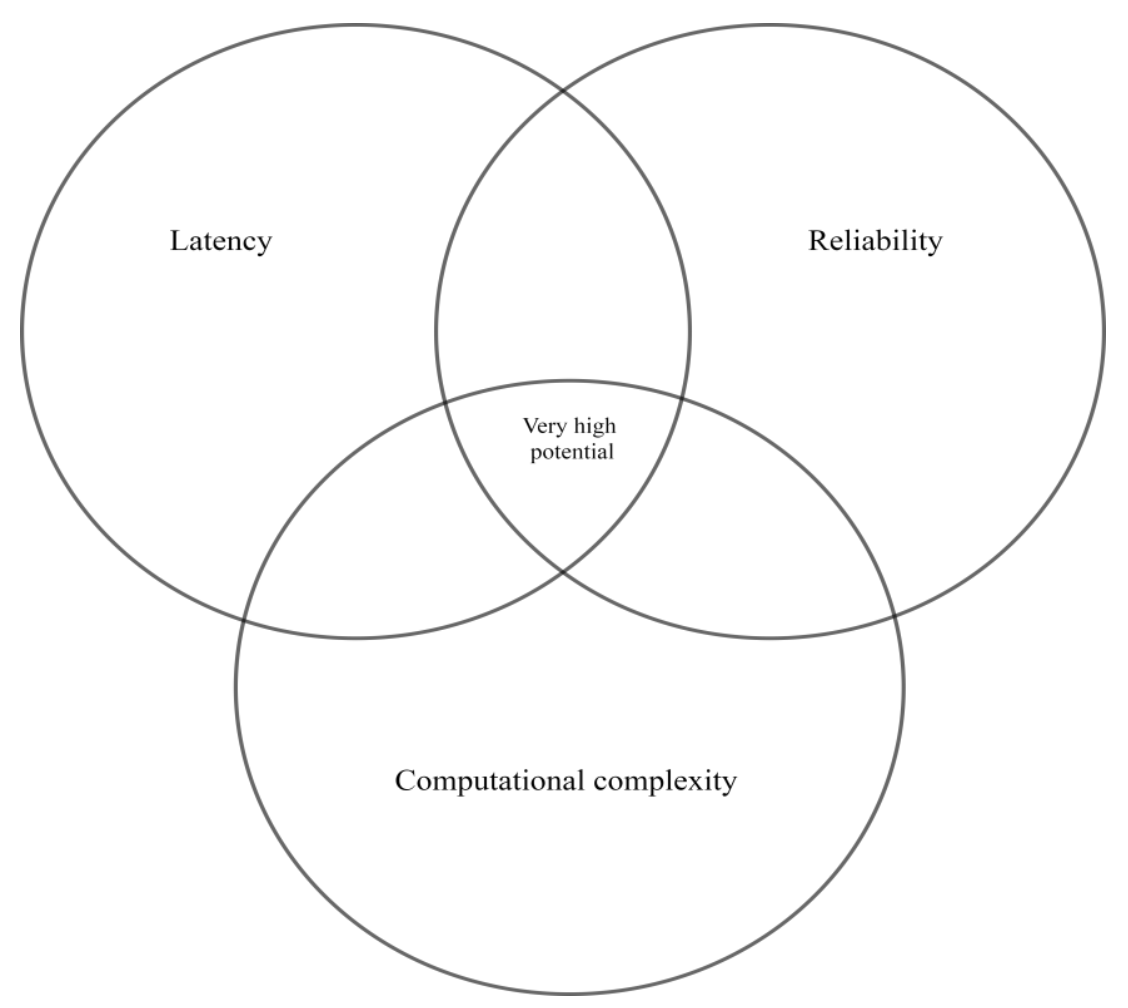

Figure 1: Requirements for the channel schemes for UWAC

In this work, different channel coding schemes, turbo, convolution, LDPC, and polar codes are considered and evaluated for application in the transmission of lengthy messages ( $256<\mathrm{K} \leq 1024$ bits) with respect to user requirement of complexity, BER and latency performance on the t-distribution of noise channel with Binary phase-shift Keying (BPSK) of code rate 1/2. Next, Section 2 presents the channel model in UWA while Section 3 describes the channel coding schemes. The results and analyses are given in Section 4 and finally, 5 concludes the research. 


\section{Channel model in UWA}

Based on the analysis of the field data measurements in a shallow water, a noise model of the underwater acoustic channel is presented in [5]. The likelihood density function has been proposed for the noise amplitude distribution and the probability functions have been derived in [5],[6]. Hence, for binary signalling of the channel, an expression to the symbol error probability is presented. The noise data collected from a shallow water was also simulated to show the noise effects on the performance of binary signalling system of UWAC. In addition, t-distribution was used to show good fitting of the field measurement analysis of the amplitude of the noise distribution with average degree of freedom of about 3. Equation (1) expresses the symbol error probability of the binary UWA noise channel.

$$
P_{B P S K}=0.636 \sqrt{\frac{2 E_{b}}{N_{o}}} \int_{0}^{\infty}\left[1+\frac{2 E_{b}}{N_{o}}(x+1)^{2}\right]^{-2} d x
$$

where $x$ is a vector of $M$ discrete amplitude levels of noise, and $b_{o} / E_{N}$ is the energy per bit to noise power spectral density ratio. Some researchers have indicated that the noise distribution in shallow water follows the Gaussian distribution while others assume the noise distribution in UWA is non-white and non-Gaussian [7],[8].

\section{Channel coding schemes}

In channel coding, redundancies are added to some useful bits to secure the data in a noisy channel and to reduce transmission errors to the minimum regardless of its intricacies in communication systems. The main step of the channel coding procedures is partitioning the coding into several subsets. There are four common kinds of the channel coding schemes and these are convolutional codes (CC), turbo codes (TC), polar codes (PC) and low density parity check (LDPC) code [9]. Figure 2 depicts the digital communication system block diagram of the different channel coding schemes. The most important aspect of channel coding is its ability to provide the satisfaction for user requirements. 


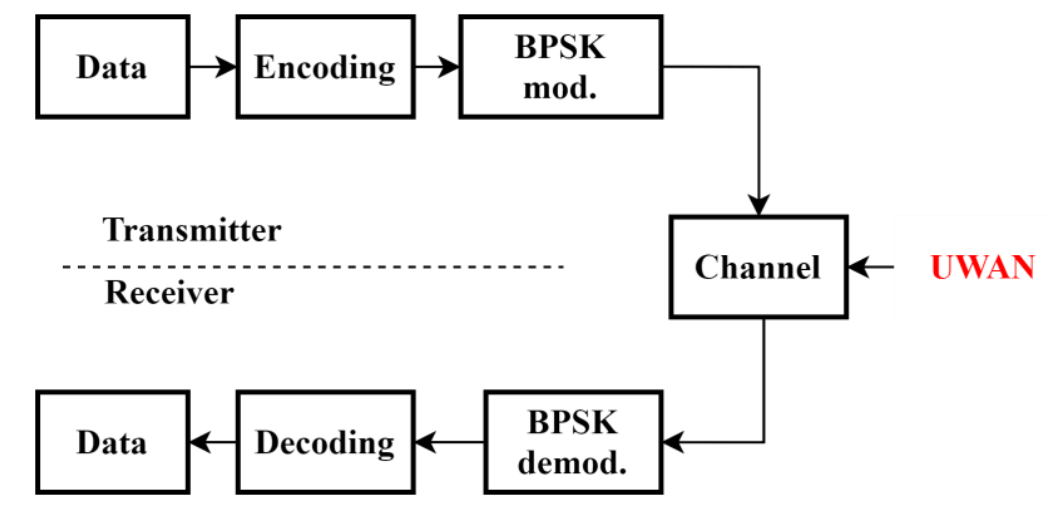

Figure 2: Digital communication system block diagram of the channel coding schemes

\section{1 convolution code}

Convolution code (CC) is a division of linear error-code broadly applicable to systems such as IS-95 based narrow band CDMA, GSM and WCDMA [10]. A set number of the shift registers is used to develop the convolutional encoder. Each of the input bits is made to enter the shift register with the encoder output obtained from the combination of the bits in the shift register. Three parameters, the output bits, $v$, input bits, $u$ and the number of memory registers, $m$ are used to define CC [11]. The code rate, $R=u / v$ gives the coding efficiency with values of $u$ and $v$ ranging from 1 to 8 and 2 to 10 , respectively. One CC defined as CC $(7,5)$ was used. Using trellis representation by employing four states following the CC order, Viterbi algorithm was utilized for the decoding.

The decoding could be soft or hard. In hard decoding, only binary values are used, but real values, generated by the output equalizer, are utilized in soft decoding. However, sequential decoding comes with a disadvantage of returning a random variable of the searched number of state metrics [12]. The increasing value of the look-ahead length of the feedback decoder not only increase the coding gain but also increases the complexity of the decoding implementation [13]. With the Viterbi algorithm, exponential decrease in the error probability with increasing number of code states, $\mathrm{S}$ and constraint length, $u$ is its disadvantage. Some research works such as those presented in [14],[15] have utilized $C C$ in underwater acoustic with u-order code implemented by several options. Figure 3 shows the best polynomial, which is arrived at by simulations, and trial and error method [16]. 


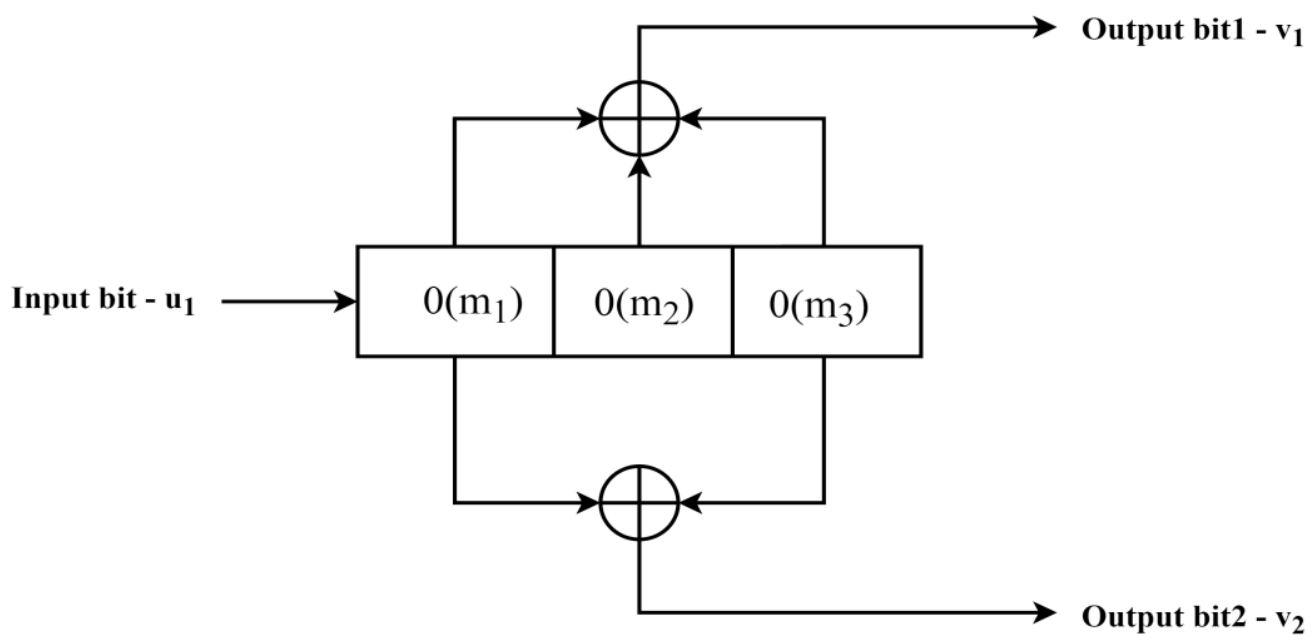

Figure 3: Convolutional encoder structure [14]

\subsection{Turbo code}

Turbo codes (TC) are normally comprised of two parallel convolution encoders separated by an interleaver [17]. The main aim is the selection of suitable interleaver by the construction of the polynomial codes for individual encoders. With the considerations of the individual encoders as convolutional, the interleaver is the main element to be analysed and discussed in the encoding process. Figure 4 shows a turbo encoder using an interleaver. A systematic stream output, $u_{t}$ and a parity stream, $b_{1}$ are contained in the first encoder (containing the systematic and the first parity stream) while only parity stream, $b_{2}$ is present in the second individual encoder (containing the interleaved systematic stream and the second parity stream) resulting to the formation of 1/3 TC. In addition, performance losses are avoided by acknowledging the first and last states of the encoder at the decoder by trellis termination. Two soft-input soft-output (SISO) decoders represents the turbo decoder at the decoding unit. It is worthy of note that both turbo decoders and the convolutional decoders are similar in structure with only a few variations. The decoding iterative scheme is essentially comprised of an interleaver, a posteriori probability (APP) decoder and deinterleaver [11]. 


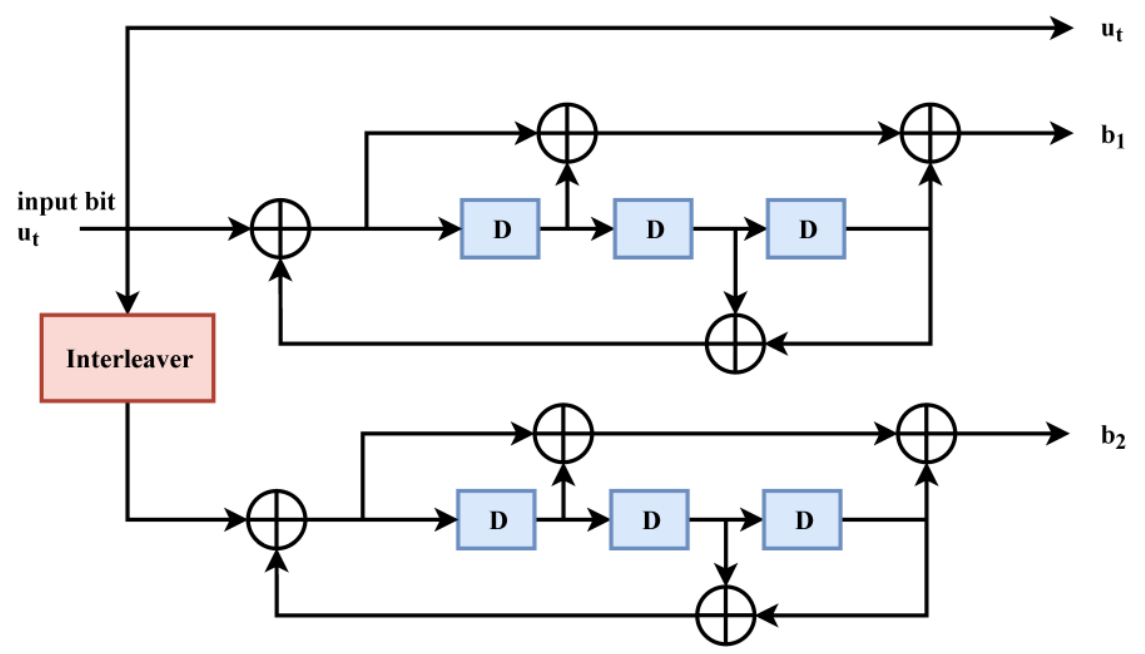

Figure 4: Turbo code structure [11]

Nevertheless, turbo coding is faced with a major challenge of characteristic delay linked with both the decoding algorithm and the interleaver [10]. The associated high implementational complexities could make the block lengths and code rates fail to satisfy the requirements of UWA [18]. Several submissions have been made on turbo codes and its use in UWA [5],[10],[19].

\subsection{LDPC code}

A class of linear error-correction code, Low-Density Parity Check (LDPC) matrix code, was in 1962, introduced by Gallager [20]. It is characterized by a sparsed parity check matrix $H$. WiMAX and DVB-S2 adopted LDPC code. LDPC code can be considered regular or irregular LDPC codes [21]. It is regular when $H$ has equal number of ones in each column, $w_{c}$ and the equal number of ones in each row, $w_{r}$ [22]. An example of regular binary LDPC codes [21] is the original Gallager codes [20]. Although, it has generally very large size of $H$ matrix, it has very low the density of the nonzero element [12]. LDPC code of length, $n$ can be written as $\left(n, w_{c}, w_{r}\right)$. Thus, each information bit is characterized by $w_{c}$ parity checks while each parity check bit is characterized by $w_{r}$ information bits [12]. An algebraic construction can be used to represent the parity check matrix, $H$ [21], and by Tanner graph [21]. In this case, the variable or bit nodes ( $\mathrm{VN}$ or $\mathrm{BN})$ in Tanner graph is equal to $w_{c}$, and the check nodes $(\mathrm{CN})$ is equal to $w_{r}$ connected by $1 \mathrm{~s}$ in the $H$ matrix. The representation of Tanner graph is shown in Figure 5. An example of $H$ matrix algebra representation is given in equation (2) [21]. 


$$
H=\left[\begin{array}{llllllllllll}
1 & 1 & 1 & 0 & 1 & 1 & 1 & 0 & 0 & 0 & 0 & 0 \\
0 & 0 & 0 & 0 & 1 & 1 & 1 & 1 & 0 & 1 & 0 & 1 \\
1 & 0 & 0 & 1 & 0 & 0 & 0 & 1 & 1 & 1 & 0 & 1 \\
0 & 1 & 0 & 1 & 0 & 0 & 1 & 1 & 1 & 0 & 1 & 0 \\
1 & 1 & 1 & 1 & 0 & 0 & 0 & 0 & 0 & 0 & 1 & 1 \\
0 & 0 & 1 & 0 & 1 & 1 & 0 & 0 & 1 & 1 & 1 & 0
\end{array}\right]
$$

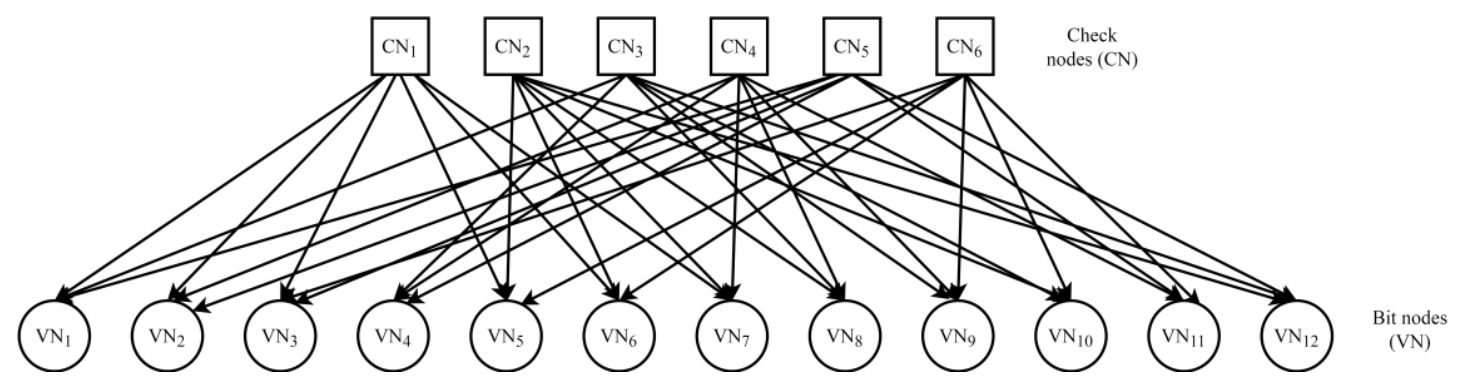

Figure 5. Tanner graph of the example $H$ matrix

LDPC is a simple code which is characterised by linear time encoding and decoding with high efficiency and low complexity compared with turbo code. LDPC code is a high-performance coding scheme of RF communication, however, it is not used in the UWAC. This is because firstly, a long package (about 1000 bits) is needed whereas the UWA communication commonly adopts a short package since the channel is characterised by variable propagation delay and low communication rate [10]. Secondly, it requires more iterations which makes it more complex as compared to other simplified codes [23].

\subsection{Polar code}

In 2009, polar codes (PC) were invented by Erdal Arikan [24]. This class of linear error-correction code was introduced based on polarization technique, a new concept. PC became active research focus as soon as they were invented. This is owned to the fact that PC demonstrate promising outcome with increasing capacity. They have shown better performance over other coding techniques and hence, extended to cover a number of applications and generalizations as presented in [25]. PC offer very low complexity and explicit construction encoding and decoding. Furthermore, arbitrary design of the code rate of a PC is possible making it more flexible for applications to various scenarios, such as security coding on the physical layer [4]. PC can be defined as $(N, K)$ with $N$ and $K$ denoting the encoded block and the information block lengths, respectively. $N=2^{n}$, and $n \geq 0$ where $\mathrm{n}$ is a natural integer [27]. The remaining $N-K$ 
indices is called the frozen bit indices [27]. Channel polarization transform is used to construct the PC [26] with the polarization transform being the encoder, which is given by the kernel of equation (3) [24].

$$
F=\left[\begin{array}{ll}
1 & 0 \\
1 & 1
\end{array}\right]
$$

For larger input size, the transform is found the Kronecker product of this kernel with itself. This causes the PC to have lengths of powers of 2 . For a code of length $N$, and $n=\log _{2}(N)$, the encoder is given by equation (4)

$$
G=F^{\otimes n}
$$

where $F^{\otimes n} F$ is the Kronecker product of $F$ with itself for $n$ times. The encoding is then carried out as in (4), which is shown in Figure 6 for a code of length 4. In this study, the technique of Successive Cancellation (SC) decoding is applied as the decoder for the PC due to the regular structure of the PC.

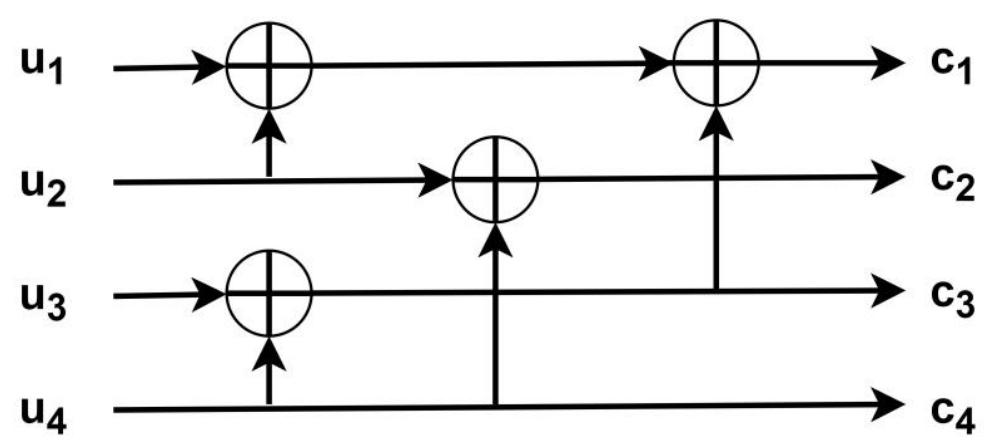

Figure 6. Polar encoder of length 4.

\section{Results and Analyses}

This section presents the outcomes of UWA system simulation to evaluate the performance of channel coding schemes based on t-distribution of the noise channel. Table 1 shows the simulation parameters applied for the communication scheme. The analysis of UWA system is evaluated in three phases. Firstly, BER reduction of CC, TC, LDPC and PC is assessed and compared with the signal to noise ratio (SNR). Secondly, the computational complexity level was analysed and lastly, the decoding latency of the channel coding schemes is investigated and discussed. The results of the simulations were retrieved from simulation in MATLAB using the adopted BPSK with code rate $1 / 2$. 
Table 1. Simulation parameters for the baseline

\begin{tabular}{|l|l|}
\hline Parameters & Values \\
\hline Modulation constellation $(\mathrm{M})$ & BPSK \\
\hline Degree of freedom $(d)$ & 3 \\
\hline Channel & t-distribution noise \\
\hline Code rate & $1 / 2$ \\
\hline Channel decoding algorithm & CC(Viterbi) TC(MAX-Log-MAP) LDPC( MSP) PC(SC) \\
\hline Message length & 256,512, and 1024 \\
\hline
\end{tabular}

\subsection{BER performance}

For UWAC system of message transmission, BER reduction is the first requirement of the channel coding. The reliability is then evaluated based on BER comparison of CC, Turbo, LDPC, and PC schemes for the UWAC. This is done for the transmission of different lengths of messages using the parameters as determined in Table 1. The simulations results for all the schemes are depicted in Figures 7 through 9. These results are compared with the uncoded schemes by theory and simulation for the length of message $(256 \leq \mathrm{K} \leq 1024$ bits) at code rate $1 / 2$. Good performance for UWA based on TC, LDPC and PC are depicted in Figure 7 where the BER of all the schemes is less than $10^{-4}$ for channels with low SNR (0 to $3 \mathrm{~dB}$ ), which notably outperforms the CC.

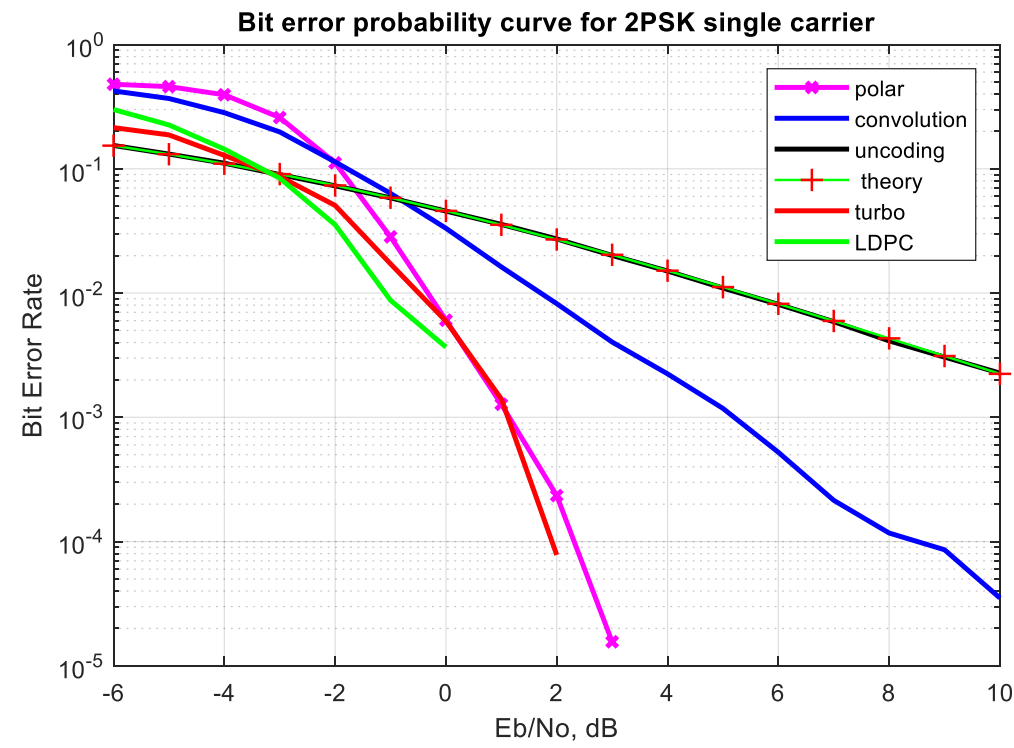

Figure 7: BER performance evaluation for different channel coding schemes $(\mathrm{K}=256$ bits) 
From Figures 8 and 9, it is clear that polar code scheme is more significant (BER performance is $10^{-3}$ at SNR $-1 \mathrm{~dB}$ ) than TC, LDPC, and CC schemes for different message lengths $(512 \leq \mathrm{K} \leq 1024$ bits) at code rate of $1 / 2$. The PC scheme has better reliability with an improvement of $1.5 \mathrm{~dB}$ channel gain compared with TC scheme at length [K =512 and 1024 bits] because the SC decoder of polar code has an acceptable performance in short length messages however, it achieves better results in longer length messages. Also, Figures 8 and 9 reveal that BER of $10^{-4}$ at message $\mathrm{K}=$ 512 and 1024 bits is achievable using the TC. Since the message length is short and TC is characterized by random interleaving, the random position in the medium is not really very random. Furthermore, the decoding capability is limited by punctured TC to correct the errors emanating from the removal of some of the parity bits.

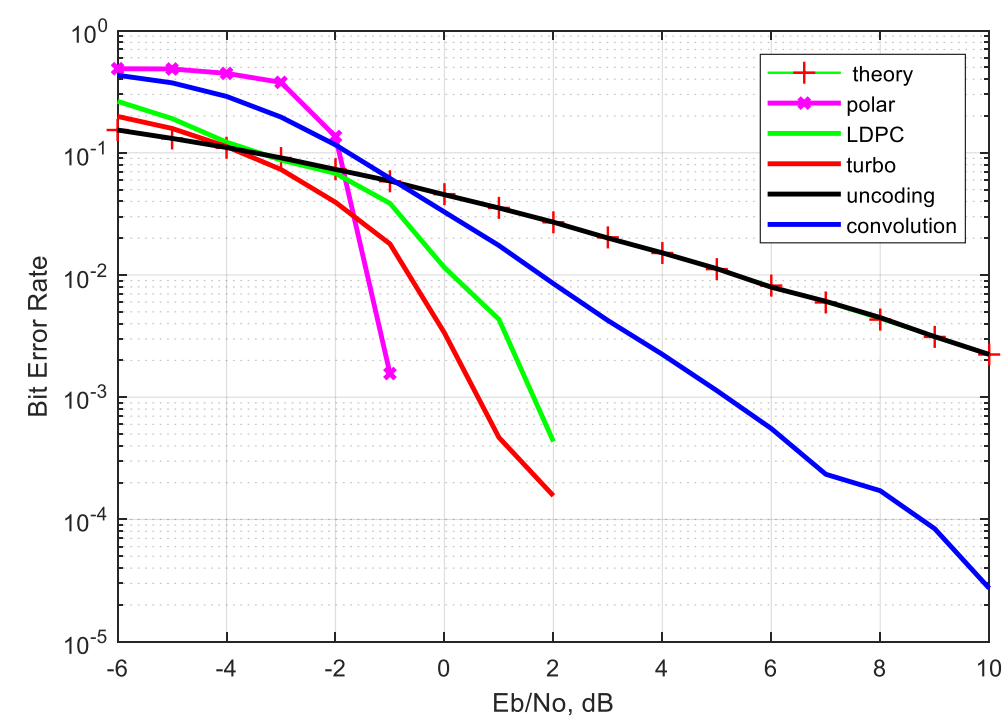

Figure 8: BER performance of different channel coding schemes for $\mathrm{K}=512$ bits 


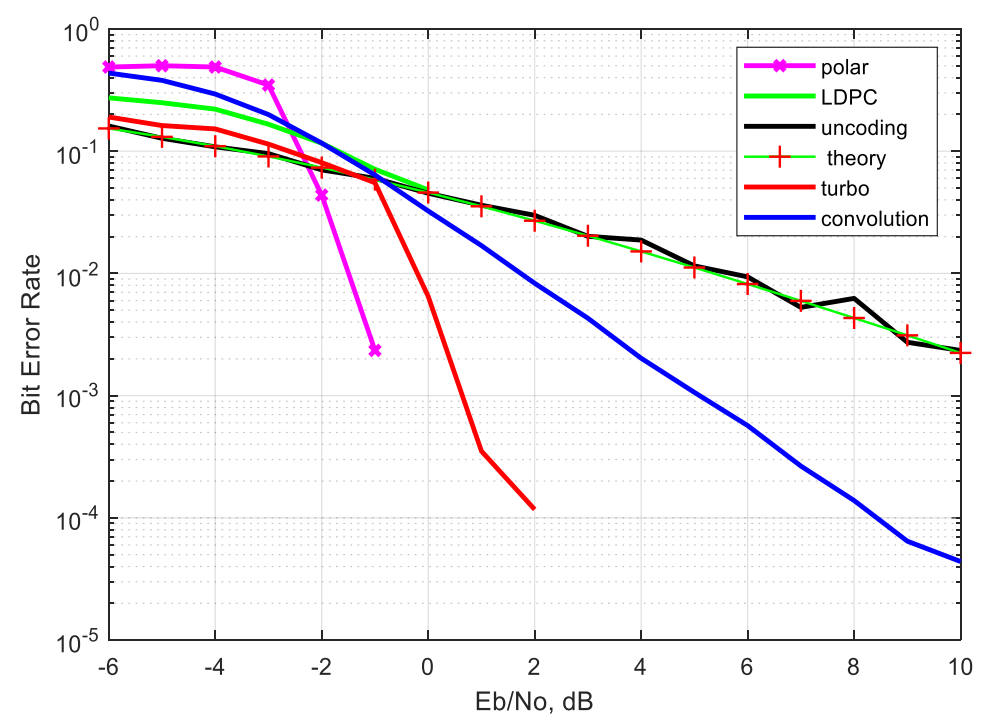

Figure 9: BER performance of different channel coding schemes for $K=1024$ bits

\subsection{Complexity}

The computational complexity is an essential performance metric to be evaluated in codes building and its applied especially in UWA. Therefore, in this paper, the complexity evaluation is analysed and discussed, which is important in examining the computational complexity in the encoding and decoding techniques. In this section, it is required to understand the complexity of the decoding algorithm used to evaluate and find the iteration number identical to providing fair comparison for different schemes. The decoding complexity can be evaluated through multiple mathematical operations such as addition, subtract, multiplication, division, and comparison. Based on code building, different result conclusions can be obtained.

The parameters related to the computational complexity of the PC scheme is calculated and compared with TC, LDPC and CC schemes. Table 2 presents the equations used in the determination of the number of operations for the various schemes. The parameters $\mathrm{K}, \mathrm{R}$ and $\mathrm{N}$ denote the information block length, the code rate and the encoded block length respectively [27] [24]. Additionally, $m$ represents the memory length of the component for TC and CC [28],[29]; $d_{c}$ and $d_{v}$ represent the average check degree and average variable degree respectively for LDPC scheme [30], while $I_{\max }$ stands for the maximum number of iterations. 
Table 2. Computational complexity of polar code, turbo code and some of the previously channel code methods in literature

\begin{tabular}{|c|c|}
\hline Method & Computational complexity \\
\hline Polar [24] & $O\left(N \log _{2}(N)\right)$ \\
\hline Turbo [27] & $I_{\max } \times 16 \times K \times 2^{m}+I_{\max } \times 8 \times K \times 2^{m}$ \\
\hline LDPC [30] & $I_{\max } \times 2 \times N \times d_{v}+M \times\left(2 \times d_{c^{-}} 1\right)+I_{\max } \times M \times d_{c}$ \\
\hline Convolution [28] & $4 \cdot R \cdot N \cdot 2^{m}$ \\
\hline
\end{tabular}

Table 3 shows the comparison of the computational complexity of each studied channel coding scheme with TC scheme. It can be seen that the computational complexity of PC scheme is kept lowest as compared with other coding schemes along with the variation of $\mathrm{K}$ parameter. This could be attributed that the PC scheme is constructed based on simple algorithm (Successive Cancellation). Therefore, it is implemented efficiently by the factor graph of the code that has a structure resembling the Fast Fourier Transform (FFT) [24]. On the other hand, the coding schemes of TC and LDPC show higher computational complexity compared with PC and CC schemes. This is because, they construct base on an iterative algorithm with high number of iterations leading to increase in the computational complexity. The comparison is clearly illustrated in Figure 10.

Table 3. comparison of the computational complexity of polar code and turbo code

\begin{tabular}{|c|c|c|c|c|c|c|c|c|c|}
\hline \multirow{2}{*}{$\begin{array}{l}\text { Code } \\
\text { Scheme }\end{array}$} & \multicolumn{7}{|c|}{ Parameters } & \multirow[b]{2}{*}{ Complexity } & \multirow{2}{*}{$\begin{array}{c}\text { Complexity Ratio } \\
\text { w.r.t. Turbo } \\
\text { decoder }(\%)\end{array}$} \\
\hline & $K$ & $m$ & $d_{c}$ & $d v$ & $\begin{array}{c}N= \\
(K / R)\end{array}$ & $\mathrm{R}$ & $I_{\max }$ & & \\
\hline Turbo & \multirow{4}{*}{256} & 3 & - & - & - & \multirow{4}{*}{$1 / 2$} & 8 & 393216 & 100 \\
\hline LDPC & & - & 7.17 & 3.58 & - & & 16 & 142663.68 & 36.28 \\
\hline $\mathrm{CC}$ & & 6 & - & - & \multirow{2}{*}{512} & & - & 65536 & 16.66 \\
\hline Polar & & - & - & - & & & - & 4608 & 1.17 \\
\hline Turbo & \multirow{4}{*}{512} & 3 & - & - & - & \multirow{4}{*}{$1 / 2$} & 8 & 786432 & 100 \\
\hline LDPC & & - & 7.17 & 3.58 & - & & 16 & 285327.36 & 36.28 \\
\hline $\mathrm{CC}$ & & 6 & - & - & \multirow{2}{*}{1024} & & - & 131072 & 16.66 \\
\hline Polar & & - & - & - & & & - & 10240 & 1.30 \\
\hline Turbo & \multirow{4}{*}{1024} & 3 & - & - & - & \multirow{4}{*}{$1 / 2$} & 8 & 1572864 & 100 \\
\hline LDPC & & - & 7.17 & 3.58 & - & & 16 & 570654.72 & 36.28 \\
\hline $\mathrm{CC}$ & & 6 & - & - & \multirow{2}{*}{2048} & & - & 262144 & 16.66 \\
\hline Polar & & - & - & - & & & - & 22528 & 1.43 \\
\hline
\end{tabular}




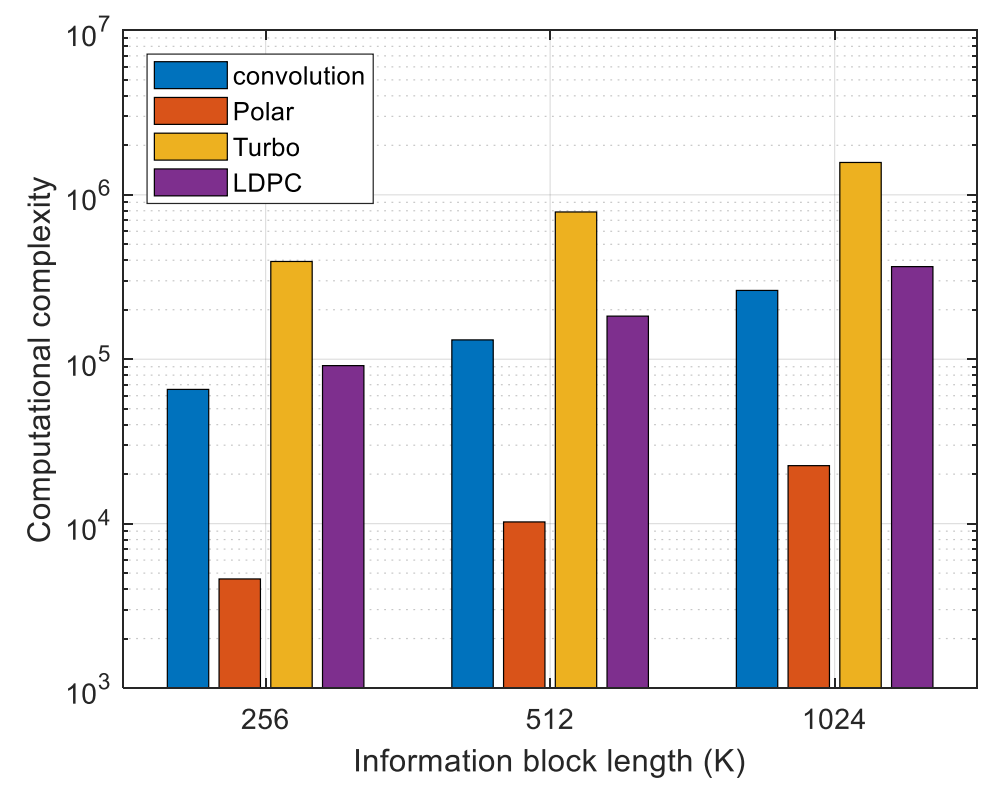

Figure 10. Computational complexity versus information block lengths, $\mathrm{K}$ for various techniques with coding rate of $\mathrm{R}=1 / 2$.

\subsection{Latency}

Latency is considered as the third requirement for the implementation of UWA communication channel coding schemes. It is defined as the time taken for the message bits transmitted over all communication blocks as displayed in Figure 2. In this section, the latency is evaluated and compared in terms of different channel decoding schemes such as polar, Turbo, LDPC and Convolution coding. The MATLAB functions tic and toc are used to calculate the decoding latency time with taking in consideration the type of processor used in the simulation. The simulation result shown in Figure 11 is the computational latency of the different channel coding schemes of UWA for transmitted message length of $256 \leq \mathrm{k} \leq 1024$ bits at code rate of $1 / 2$. The PC gives higher decoding computational latency compared with the other coding schemes as a result of the serial decoding by SC algorithm. It is timeconsuming and could get worse with an increasing code length, N. 


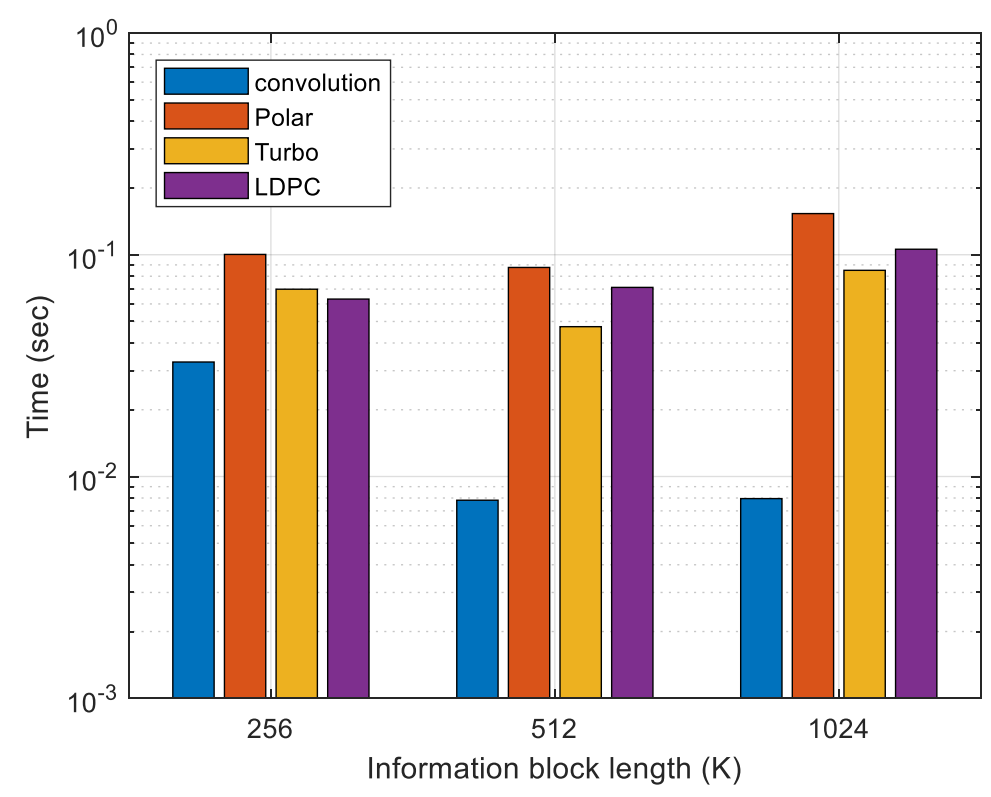

Figure 11. Decoding computational latency

Figure 11 also shows TC (MAX-Log-MAP) and LDPC (MSP) give have a higher decoding computational latency than $\mathrm{CC}$ (Viterbi) owing to the associated iterative algorithms. Higher decoding computational latency is given by the LDPC (MSP) compared with the TC (MAX-Log-MAP) since LDPC (MSP) requires more number of iterations $\left(I_{\max }=16\right)$ than the TC $\left(I_{\max }=8\right)$. However, if the system can tolerate large time delay and complexity, TC will be considered one of the competing error correction codes.

\section{Conclusion}

In conclusion, different channel coding schemes of convolution, Turbo, LDPC, and Polar coding were analysed for UWA system based on the t-distribution noise channel with BPSK modulation. The evaluated results of the different coding schemes show that polar coding is the optimum channel coding scheme for UWA as it fulfils user requirements in terms of BER reduction and low computational complexity. Moreover, other requirements such as application type, quality, cost, and data transmission rate are often considered in the choice of channel coding techniques. For instance, the high-speed data rate requires low computational complexity, whereas an excellent BER reduction performance is needed for high-quality. Hence, there i9s always a trade-off between computational complexity and BER reduction 
performance in the channel coding techniques. This must be kept firmly considered in the choice of channel coding technique for BER reduction.

Acknowledgements This research was funded by the Ministry of Higher Education

Malaysia under Fundamental Research Grant Scheme Vot No. K096 and partially sponsored by Universiti Tun Hussein Onn Malaysia.

\section{References}

[1] M. F. Ali, D. N. K. Jayakody, Y. A. Chursin, S. Affes, and S. Dmitry, "Recent advances and future directions on underwater wireless communications," Archives of Computational Methods in Engineering, pp. 1-34, 2019.

[2] A. Song, M. Stojanovic, and M. Chitre, "Editorial Underwater Acoustic Communications: Where We Stand and What Is Next?," IEEE Journal of Oceanic Engineering, vol. 44, pp. 1-6, 2019.

[3] M. S. Ahmed, N. S. Mohd Shah, Y. Y. Al-Aboosi, M. S. Gismalla, M. F. Abdullah, Y. A. Jawhar, et al., "Filter orthogonal frequency-division multiplexing scheme based on polar code in underwater acoustic communication with non-Gaussian distribution noise," ETRI Journal, vol. 43, pp. 184-196, 2021.

[4] G. Qiao, S. Xing, and F. Zhou, "A Multi-User Detection Scheme Based on Polar Code Construction in Downlink Underwater Acoustic OFDM Communication System," IEEE Access, vol. 7, pp. 65973-65981, 15 May 20192019.

[5] M. S. Ahmed, N. S. M. Shah, F. Ghawbar, Y. A. Jawhar, and A. A. Almohammedi, "Filtered-OFDM with channel coding based on T-distribution noise for underwater acoustic communication," Journal of Ambient Intelligence and Humanized Computing, pp. 1-14, 2020.

[6] N. S. M. Shah, Y. Y. Al-Aboosi, and M. S. Ahmed, "Error Performance Analysis in Underwater Acoustic Noise With Non-Gaussian Distribution," TELKOMNIKA, vol. 16, pp. 681-689, 2018.

[7] Y. Y. Al-Aboosi, A. Z. Sha'ameri, and A. H. Sallomi, "Enhancement signal detection in underwater acoustic noise using level dependent estimation time-frequency denoising technique," Journal of Marine Engineering \& Technology, vol. 19, pp. 1-14, 2020.

[8] Y. Y. Al-Aboosi, M. S. Ahmed, N. S. M. Shah, and N. H. H. Khamis, "STUDY OF ABSORPTION LOSS EFFECTS ON ACOUSTIC WAVE PROPAGATION IN SHALLOW WATER USING DIFFERENT EMPIRICAL MODELS," vol. 12, pp. 6474-6478, NOVEMBER 20172017. 
[9] J. Huang, S. Zhou, and P. Willett, "Nonbinary LDPC coding for multicarrier underwater acoustic communication," IEEE Journal on Selected Areas in Communications, vol. 26, 2008.

[10] L. Liu, Y. Wang, L. Li, X. Zhang, and J. Wang, "Design and implementation of channel coding for underwater acoustic system," in ASIC, 2009. ASICON'09. IEEE 8th International Conference on, 2009, pp. 497-500.

[11] B. Tahir, S. Schwarz, and M. Rupp, "BER comparison between Convolutional, Turbo, LDPC, and Polar codes," in Telecommunications (ICT), 2017 24th International Conference on, 2017, pp. 1-7.

[12] Z. R. M. Hajiyat, A. Sali, M. Mokhtar, and F. Hashim, "Channel Coding Scheme for 5G Mobile Communication System for Short Length Message Transmission," Wireless Personal Communications, vol. 106, pp. 377-400, 2019.

[13] B. Sklar, "Digital Communications Fundamentals and Applications," Instructor, vol. 201705, 2017.

[14] J. Trubuil, A. Goalic, and N. Beuzelin, "An overview of channel coding for underwater acoustic communications," in MILITARY COMMUNICATIONS CONFERENCE, 2012-MILCOM 2012, 2012, pp. 1-7.

[15] P. Zhu, X. Xu, X. Tu, Y. Chen, and Y. Tao, "Anti-Multipath Orthogonal Chirp Division Multiplexing for Underwater Acoustic Communication," IEEE Access, vol. 8, pp. 13305-13314, 2020.

[16] S. Bernard, "Digital communications fundamentals and applications," Prentice Hall, USA, 2001.

[17] C. Berrou, A. Glavieux, and P. Thitimajshima, "Near Shannon limit error-correcting coding and decoding: Turbo-codes. 1," in Communications, 1993. ICC'93 Geneva. Technical Program, Conference Record, IEEE International Conference on, 1993, pp. 1064-1070.

[18] L. Liu, Y. Zhang, P. Zhang, L. Zhou, and J. Niu, "Channel coding for underwater acoustic single-carrier CDMA communication system," in Seventh International Conference on Electronics and Information Engineering, 2017, p. 103222S.

[19] M. Falk, G. Bauch, and I. Nissen, "On Channel Codes for Short Underwater Messages," Information, vol. 11, p. 58, 2020.

[20] R. G. Gallager, "Low-density parity-check codes," IRE Transactions on information theory, vol. 8, pp. 21-28, 1962.

[21] W. Han, J. Huang, and M. Jiang, "Performance analysis of underwater digital speech communication system based on LDPC codes," in Industrial Electronics and Applications, 2009. ICIEA 2009. 4th IEEE Conference on, 2009, pp. 567-570. 
[22] M. El-Mahallawy, A. S. TagEldien, and S. S. Elagooz, "Performance enhancement of underwater acoustic OFDM communication systems," Wireless Personal Communications, vol. 108, pp. 2047-2057, 2019.

[23] J. Malhotra, "Investigation of channel coding techniques for high data rate mobile wireless systems," International Journal of Computer Applications, vol. 115, 2015.

[24] E. Arikan, "Channel polarization: A method for constructing capacity-achieving codes for symmetric binary-input memoryless channels," IEEE Transactions on information Theory, vol. 55, pp. 3051-3073, 2009.

[25] W. Wang, L. Li, and K. Niu, "An efficient construction of polar codes based on the general partial order," EURASIP Journal on Wireless Communications and Networking, vol. 2019, pp. 1-12, 2019.

[26] H. Vangala, E. Viterbo, and Y. Hong, "A comparative study of polar code constructions for the AWGN channel," arXiv preprint arXiv:1501.02473, 2015.

[27] S. Shao, P. Hailes, T.-Y. Wang, J.-Y. Wu, R. G. Maunder, B. M. Al-Hashimi, et al., "Survey of Turbo, LDPC and Polar Decoder ASIC Implementations," IEEE Communications Surveys \& Tutorials, vol. 21, pp. 2309 - 2333, 2019.

[28] M. Sybis, K. Wesolowski, K. Jayasinghe, V. Venkatasubramanian, and V. Vukadinovic, "Channel coding for ultra-reliable low-latency communication in 5G systems," in 2016 IEEE 84th vehicular technology conference (VTC-Fall), 2016, pp. $1-5$.

[29] C. Berrou, A. Glavieux, and P. Thitimajshima, "Near Shannon limit error-correcting coding and decoding: Turbo-codes. 1," in Proceedings of ICC'93-IEEE International Conference on Communications, 1993, pp. 1064-1070.

[30] S.-Y. Chung, G. D. Forney, T. J. Richardson, and R. Urbanke, "On the design of lowdensity parity-check codes within $0.0045 \mathrm{~dB}$ of the Shannon limit," IEEE Communications letters, vol. 5, pp. 58-60, 2001. 